\title{
Leber's hereditary optic neuropathy: mitochondrial and biochemical studies on muscle biopsies
}

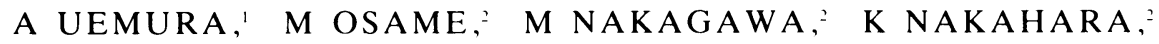 \\ M SAMESHIMA,' AND N OHBA'
}

From the Departments of 'Ophthalmology and 'Medicine, Kagoshima University Faculty of Medicine, Kagoshima-shi, Japan

SUMMARY Two patients with Leber's hereditary optic neuropathy underwent morphological and biochemical investigations of muscle biopsy samples from the biceps brachii. Although clinically there was no muscle weakness or atrophy, specific histochemical and electron microscopic examinations showed mild but distinct myopathic changes, including abnormal oxidative enzyme activities, aggregates of enlarged mitochondria in the subsarcolemmal regions, and disruptions of myofilaments. Biochemical analyses of mitochondria isolated from muscle samples did not show any defic:ency in respiratory-chain enzyme complexes or defect in content of cytochromes. Leber's hereditary optic neuropathy is assumed to be a multisystem disorder involving skeletal muscle also.

Leber's hereditary optic neuropathy is a rare condition characterised by rapid loss of central vision presenting as acute optic neuritis. Both eyes are involved, with or without an interval, and visual prognosis is usually poor owing to secondary optic atrophy. The disease generally affects healthy young adults, though mild neurological symptoms and signs are occasionally present. The mode of inheritance long remained a puzzle, but detailed pedigree analyses in recent decades have suggested a nonMendelian cytoplasmic form of inheritance. The aetiology is still uncertain, but abnormal phenotypic expression of the gene is thought to require an interaction with yet undefined extrinsic or environmental factors. ${ }^{1.5}$

Recent evidence for maternal transmission of a human mitochondrial genome prompted us to study muscle biopsy specimens obtained from patients with Leber's hereditary optic neuropathy with reference to the morphology and biochemistry of muscle mitochondria. This paper reports preliminary results indicating mitochondrial changes associated with mild myopathic processes.

\section{Materials and methods}

Two patients with Leber's hereditary optic neuro-

Correspondence to Dr Akinori Uemura, Department of Ophthalmology, Kagoshima University Faculty of Medicine, Usuki-cho 1208-1, Kagoshima-shi, 890, Japan. pathy were subjects for morphological and biochemical studies of muscle biopsies.

\section{CASE REPORTS}

Case 1. A 16-year-old male, a healthy high school student, had a sudden blurring of the left vision with no preceding illness, when he was said to suffer from acute optic neuritis. Four months later he experienced an acute blurring of the right vision and was referred to us for investigations. On examination the corrected visual acuity was 0.04 OD and finger counting at $10 \mathrm{~cm}$ OS, with dense central scotomas. Motilities, ocular anterior segments, and media were normal except for sluggish pupillary reactions. Ophthalmoscopy revealed hyperaemia and swelling of the right optic disc and temporal pallor of the left optic disc, with otherwise no remarkable change. He was given a large dose of corticosteroids, and in a few weeks the acute inflammatory changes of the right optic disc subsided but the vision did not recover. The bilateral severe visual loss with large central scotomas remained during over six months' follow-up. There was a definite family history of optic nerve disease. A brother was examined and found to have suffered from bilateral poor vision due to optic atrophy, reporting that he had experienced episodic visual loss in childhood. The parents were healthy and not consanguineous.

Case 2. A 19-year-old male was referred to us for investigation of bilateral poor vision since the age of 
15 years. At that time he had sudden loss of vision without preceding general illness in the right eye initially and with intervals of nine months in the left eye. On examination the corrected visual acuity was $0.02 \mathrm{OD}$ and $0.01 \mathrm{OS}$, with sluggish pupillary responses and large central scotomas. Motilities were normal, and the ocular anterior segments and media were also normal. Ophthalmoscopy revealed generalised pallor of both optic nerve heads. There was a family history of optic nerve disease; the patient's mother and a maternal uncle had long suffered from bilateral visual loss due to optic atrophy following acute optic neuritis.

Neither patients had any other symptoms than the visual failure. Physical and routine laboratory studies, including examination of the cerebrospinal fluid, gave normal results. Neurological examinations revealed no abnormality. In case 1 the forearm exercise test showed a normal rise of blood lactate and pyruvate levels.

\section{MORPHOLOGY AND BIOCHEMISTRY OF MUSCLE} BIOPSIES

Muscle biopsies were obtained under local anaesthesia from the left biceps brachii muscle, in case 1 during the acute inflammatory phase of the disease and in case 2 at the atrophic stage. Separate samples were processed for histochemistry, electron microscopy, and mitochondrial biochemistry.

For the histochemical and histological studies, the muscle biopsy specimens were rapidly frozen, and cryostat sections were processed with a battery of histochemical stains and examined with a light microscope. Morphological details were studied with haematoxylin and eosin and the Gomori modified trichrome stains. Mitochondrial oxidative enzyme activity was examined by the NADH-tetrazolium reductase reaction (NADH-TR). Other histochemical studies included the adenosine triphosphatase (ATPase), acid phosphatase, oil red 0, and periodic acid Schiff (PAS).

For the electron microscopic studies the muscle samples were fixed in 3\% glutaraldehyde in $0.125 \mathrm{~m}$ cacodylate buffer, $\mathrm{pH} 7 \cdot 4$, for $2 \mathrm{~h}$, rinsed in the cacodylate buffer for $1 \mathrm{~h}$, postfixed with $1 \%$ osmium tetroxide for $1 \mathrm{~h}$, dehydrated in a graded series of ethanol, and embedded in Epon 812. Ultrathin sections were stained with uranyl acetate and lead citrate, and examined with a JEOL 100-B electron microscope.

For the biochemical analysis mitochondria were isolated from frozen muscle biopsies by the method of Bookelman et al. ${ }^{6}$ Mitochondrial respiratory enzyme complexes were assayed by measuring NADHdehydrogenase, rotenone-sensitive NADH cytochrome $\mathrm{c}$ reductase, succinate cytochrome $\mathrm{c}$ reductase, cytochrome c oxidase, and citrate synthase. Reduced-minus oxidised spectra of cytochromes were recorded at room temperature in a Shimazu UV-3000 dual wave spectrophotometer. Cytochromes were reduced by addition of $1 \mathrm{mM} \mathrm{KCN}$. The concentrations of cytochromes were calculated from the extinction coefficients of Bookelman et al. ${ }^{\circ}$ Protein concentrations in mitochondrial preparations were measured by the method of Lowry et al. ${ }^{\prime}$

\section{Results}

MUSCLE HISTOCHEMISTRY

Mild features of myopathic processes were observed in muscle biopsies of the two cases. The muscle fibres measured 40 to $60 \mu \mathrm{m}$ in cross section. There was a normal reaction to the ATPase, which revealed the usual proportion of two fibre types randomly dis-
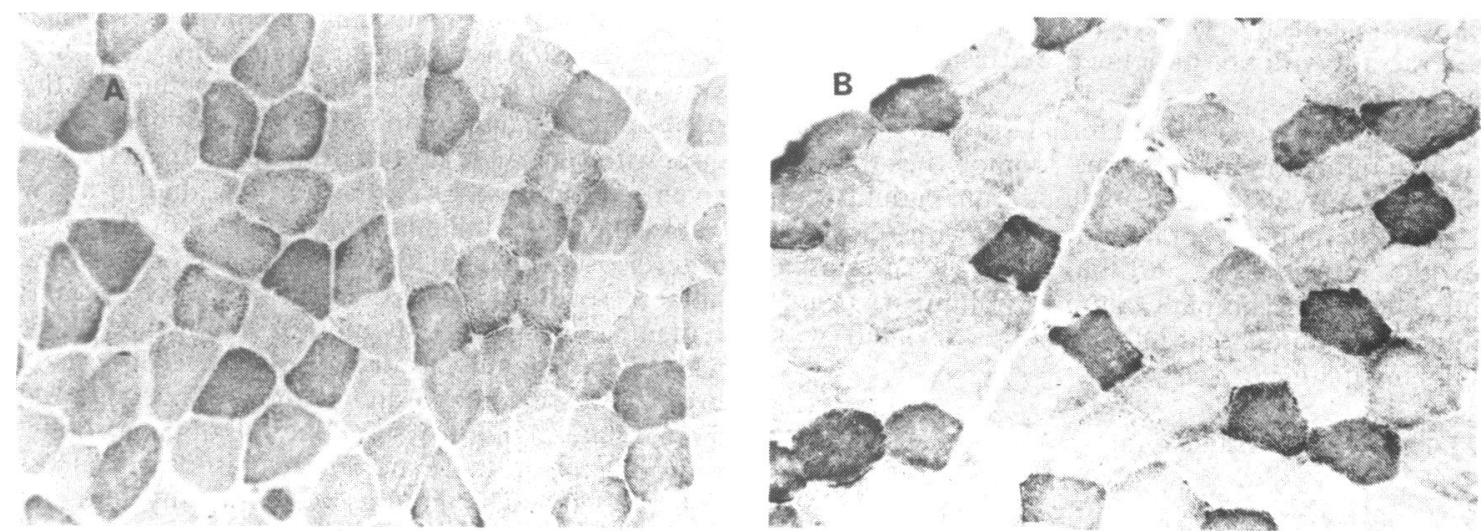

Figs. 1 (a) and (b) Muscle biopsy: cryostat sections stained with NADH-TR, illustrating focal increase of oxidative enzyme activity in many type I fibres. No other abnormality is present with two fibre types distributed in a checkerboard pattern. A: case I. B: case 2 . 
tributed in a checkerboard pattern. There was no increase in numbers of internal nuclei or increase of the endomysial connective tissue. With the modified trichrome stain a minimal amount of haematoxylinpositive material reacting red was scattered in the subsarcolemmal regions. With the NADH-TR stain numerous type I fibres showed either a focal increase or decrease in oxidative enzyme activity (Figs. 1A, B). The intracellular glycogen content demonstrable with the PAS stain was normal. In addition in case 1 there was a moderate increase in the intracellular lipids demonstrable with the oil red $\mathrm{O}$. In case 2 there was a mild increase in the acid phosphatase reaction.

\section{MUSCLE ELECTRON MICROSCOPY}

Electron microscopic examination of the muscle biopsies showed mild myopathic changes in the two cases. In case 1 the central area of some muscle cells was involved by disruption of myofilaments, with $\mathrm{Z}$ band streaming, resembling central core disease. A few muscle fibres had nemaline rods which showed the characteristic periodicities arranged in a rectangular lattice. The sarcoplasm of occasional fibres contained cytoplasmic bodies consisting of unusual deposits characterised by a central dark region containing $\mathrm{Z}$ band material and a peripheral zone made up of thin filaments extending outwards in a ray-like fashion (Fig. 2). Large numbers of enlarged mitochondria were clustered beneath the sarcolemma of some fibres (Fig. 3). Similar myopathic changes were observed in case 2, with noticeable subsarcolemmal collections of enlarged mitochondria and focal disruptions of myofilaments. In addition a few fibres had tubular aggregates beneath the sarcolemma (Fig. 4).

\section{MITOCHONDRIAL BIOCHEMISTRY}

Quantitative assays of respiratory-chain enzyme complexes in isolated mitochondria were carried out in the two cases, and the results are illustrated in Table 1 , in which control data from 10 normal subjects are also tabulated. In case 1 NADH dehydrogenase, rotenone-sensitive NADH, cytochrome c reductase, succinate cytochrome c reductase, and citrate synthase were markedly increased by twofold to threefold of the mean value of the normal, with a

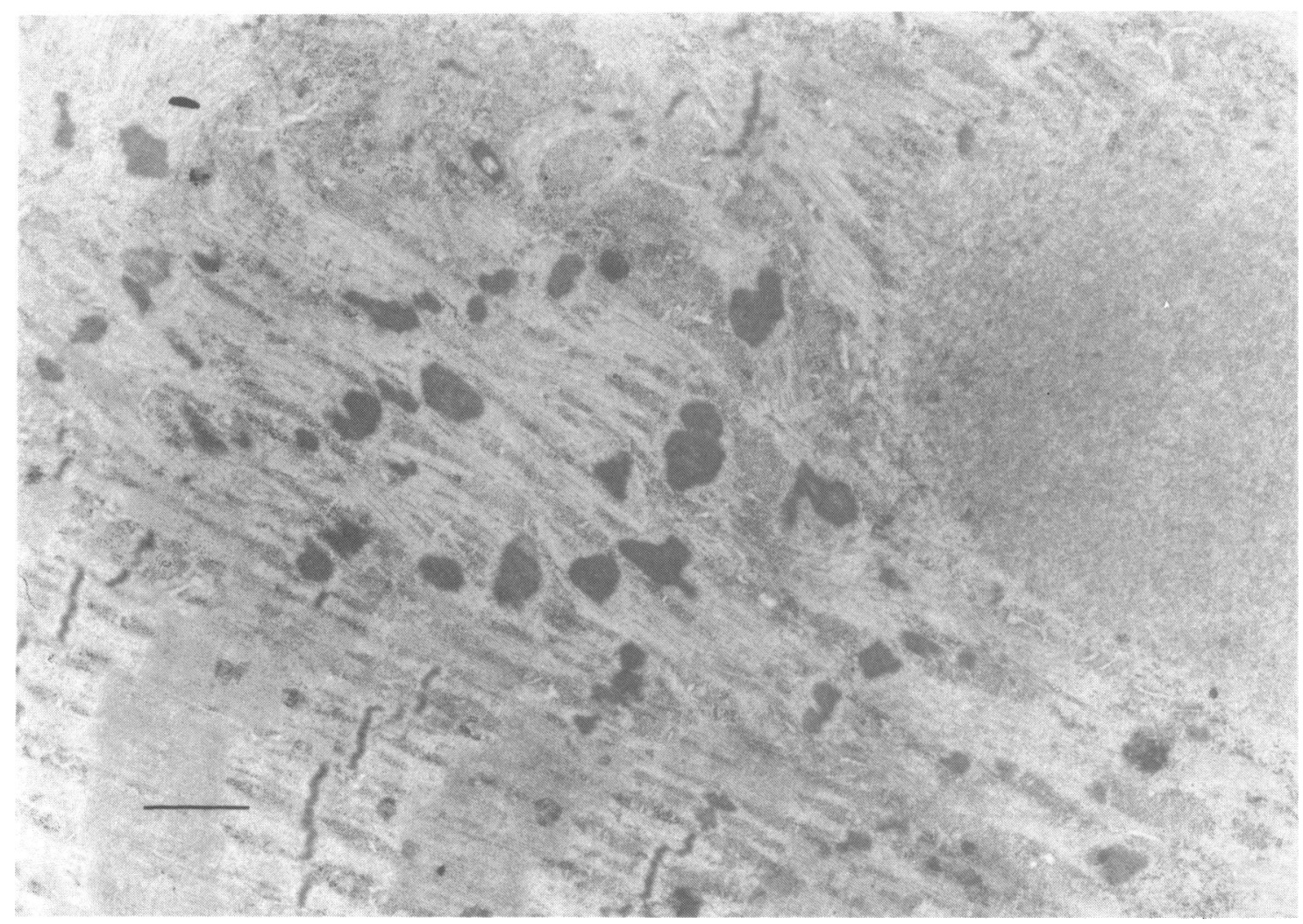

Fig. 2 Muscle biopsy (case I): electron micrograph illustrating presence of nemaline rod and cytoplasmic body with disrupted myofilaments. Bar $=1 \mu \mathrm{m}$. 
Fig. 3 Muscle biopsy (case 1): electron micrograph illustrating aggregates of enlarged mitochondria in the subsarcolemmal region. $B a r=I \mu m$.
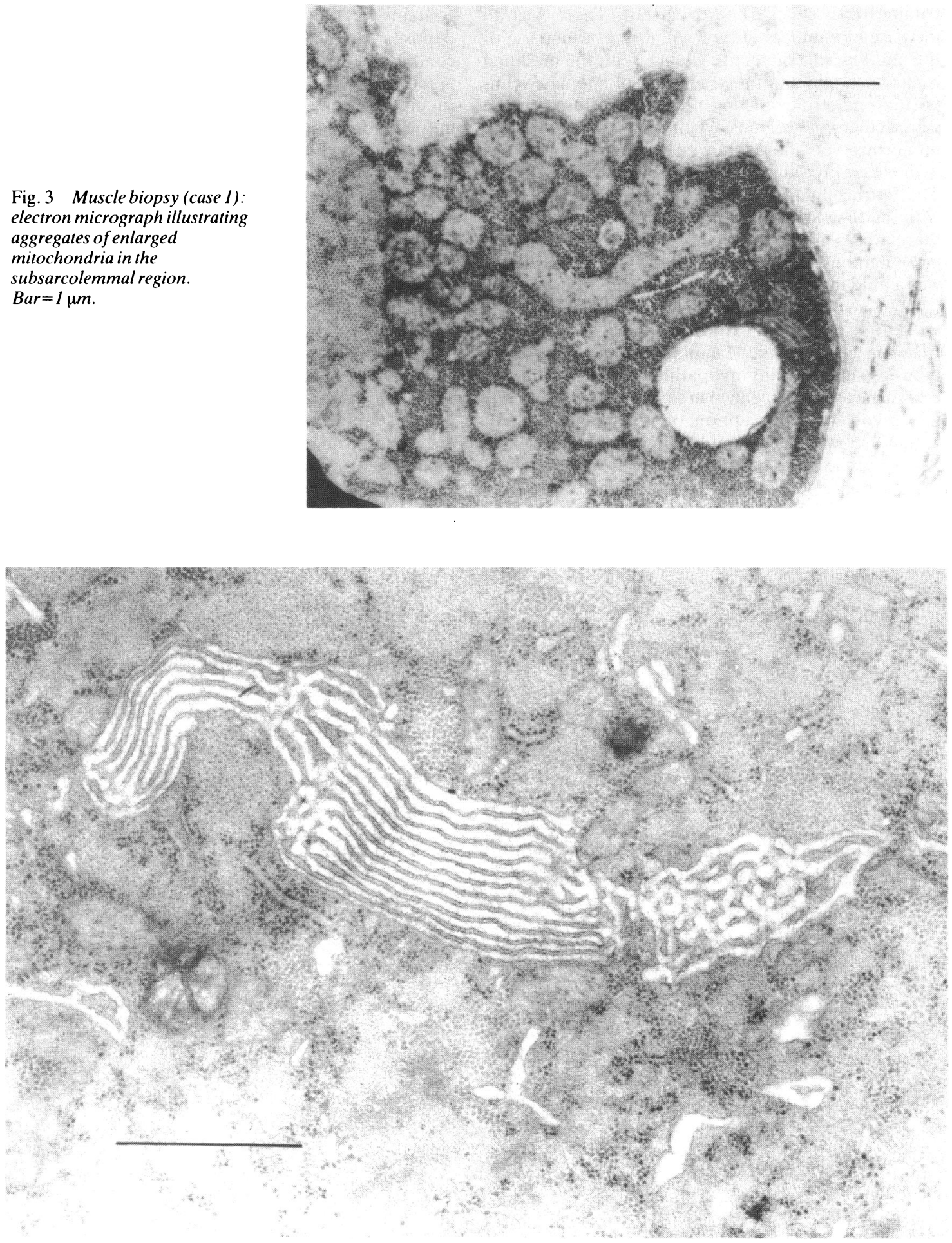

Fig. 4 Muscle biopsy (case 2): electron micrograph illustrating tubular aggregates in the sarcolemma. Bar=I $\mu m$. 
Table 1 Enzyme activity in muscle mitochondria*

\begin{tabular}{llll}
\hline Enzymes & Case 1 & Case 2 & Controls $t$ \\
\hline NADH dehydrogenase & 5.59 & 2.49 & $2 \cdot 11 \pm 1.58$ \\
NADH cytochrome c reductase & 0.431 & $0 \cdot 167$ & $0.201 \pm 0.098$ \\
Succinate cytochrome c reductasc & 1.02 & 0.357 & $0.321 \pm 0.118$ \\
Cytchrome coxidase & 1.58 & 1.85 & $1.58 \pm 0.800$ \\
Citrate synthase & 1.39 & 0.298 & $0.380 \pm 0.147$
\end{tabular}

*Expressed as $\mu \mathrm{mol} / \mathrm{min} / \mathrm{mg}$ mitochondrial protein.

†Mean \pm SD in 10 normal subjects.

$\ddagger$ Rotenone sensitive activity determined as differential activity in

the presence and absence of rotenone in the reactive mixture.

Table 2 Content of cytochromes in muscle mitochondria*

\begin{tabular}{clll}
\hline Cytochromes & Case 1 & Case 2 & Controls $\dagger$ \\
\hline$a+a_{3}$ & 659 & 519 & $550 \pm 167$ \\
$b$ & 668 & 511 & $504 \pm 122$ \\
$c+c_{1}$ & 686 & 852 & $615 \pm 258$ \\
\hline
\end{tabular}

*Expressed as pmol/mg mitochondrial protein.

†Mean \pm SD in 10 normal subjects.

normal level of cytochrome $\mathrm{c}$ oxidase. In case 2 all these enzymes were within the normal range.

The contents of mitochondrial cytochromes calculated from reduced-minus oxidised spectra are illustrated in Table 2. It can be seen that both cases had a normal concentration of mitochondrial cytochromes.

\section{Discussion}

The clinical picture in these cases, together with the familial occurrence of the disease, is in all respects consistent with Leber's hereditary optic neuropathy. The morphological studies of muscle biopsy specimens showed mild but distinct myopathic changes in the two cases examined, in that some of the fibres had noticeably abnormal reactions to a battery of histochemical stains - in particular the modified trichrome and NADH-TR-and also ultrastructural changes including nemaline rods, cytoplasmic bodies, and subsarcolemmal collections of enlarged mitochondria. These histochemical and electron microscopic abnormalities are found in case of congenital or acquired myopathies of various causes but not in normal persons. ${ }^{x \varphi}$

A variety of neurological and psychiatric symptoms and signs, including spasticity, gait disturbance, abnormal tendon reflexes, and hearing loss, are known to occur in association with Leber's optic neuropathy. Post-mortem studies also reveal widespread involvement of the central nervous system. These systemic disorders are usually so mild that they remain asymptomatic or unrecognised unless looked for specifically, though overt manifestations resembl- ing arachnoiditis or multiple sclerosis may occur in occasional patients. ${ }^{1.5}$ The present observations indicate involvement of the skeletal muscle as part of the disease, characterised by mild myopathic processes that are evident histologically but not clinically. This previously unreported findings favours the view that Leber's optic neuropathy is not confined to the optic nerve but represents a multisystem disease. ${ }^{45}$ The condition might be termed optico-encephalomyopathy.

The most notable feature of Leber's optic neuropathy is its unique hereditary pattern. There is now a fair amount of evidence suggesting that inheritance follows not Mendelian law but a pattern compatible with mitochondrial transmission. There are no recorded cases of paternal transmission, and the majority of daughters-virtually all daughters in thoroughly studied families - of patients and carriers become carriers. These phenomena are reasonably explained by maternal transmission. ${ }^{11+12}$ Furthermore, there is recent evidence for maternal transmission of the human mitochondrial genome, and many subunits of respiratory-chain enzyme complexes have been shown to be encoded by mitochondrial DNA. It is therefore possible to develop a working hypothesis that several diseases with non-Mendelian maternal transmission pattern result from inheritance of an abnormal mitochondrial genome..$^{1314}$

Two points in the present observations warrant discussion in line with the above hypothesis. First, type I fibres showed red staining material reacting to the modified trichrome stain in the subsarcolemmal regions, and the stained areas appeared to contain clusters of abnormally enlarged mitochondria at the electron microscopic level. These pathological features, together with the focal increase of oxidative enzyme activity revealed by the NADH-TR stain, are comparable to the ragged red fibres which have been known to occur in mitochondrial myopathy or mitochondrial cytopathy, a diverse group of muscle and/ or nerve diseases characterised by structurally or numerically abnormal mitochondria and/or abnormally functioning mitochondria. ${ }^{15}{ }^{16}$ Furthermore, the enlarged mitochondria in the subsarcolemmal regions may conform to the recent report that subsarcolemmal mitochondrial profiles are significantly larger in cases of Leber's optic neuropathy than in control cases. ${ }^{17}$ It seems that myopathic changes characterised by the ragged red fibres are considerably less prominent in Leber's hereditary optic neuropathy than in typical mitochondrial cytopathy. Secondly, as regards the functional aspects of muscle mitochondria in Leber's optic neuropathy, no deficiency of cytochromes or respiratory-chain enzyme complexes was detectable in the two cases examined. Rather, case 1 showed two- to threefold enhancement of the mito- 
chondrial enzyme complexes. One possible explanation for the apparently enlarged and hyperactive mitochondria is a compensation for yet undefined metabolic defect. It should, however, be emphasised that the present results do not entirely exclude the possibility of a deficiency of many other mitochondrial DNA-encoded enzymes or proteins.

The pathogenesis of Leber's hereditary optic neuropathy is unknown, but a fault in cyanide metabolism has been suggested. Recently a pronounced reduction in the activity of the enzyme thiosulphate sulphur transferase (rhodanese) was reported in liver samples from patients with the condition. $^{1 \mathrm{x}}$ This mitochondrial matrix enzyme detoxifies cyanide by a reaction with thiosulphate to form thiocyanate and sulphate. However, this finding has not been confirmed in muscle samples from patients with Leber's syndrome. ${ }^{17}$

Further studies on mitochondrial pathology in Leber's hereditary optic neuropathy are needed to elucidate its underlying metabolic defect.

\section{References}

1 Duke-Elder S, Scott GI. Leber's hereditary optic atrophy. In: Duke-Elder S, ed. System of ophthalmology. London: Kimpton, 1971; 12: 108-16.

2 Went LN. Leber's disease and variants. In: Vinken PJ, Bruyn GW, cds. Handbook of clinical neurology. Amsterdam: NorthHolland, 1972; 13: 94-110.

3 Imachi J. Leber's discasc. Acta Soc Ophthalmol Jpn 1973; 77: 1658-735.
4 Wilson J. Leber's hereditary optic atrophy: some clinical and actiological considerations. Brain 1963; 86: 347-62.

5 deWeerdt CJ, Went LN. Neurological studies in families with Leber's optic atrophy. Acta Neurol Scand 1971; 47: 541-54.

6 Bookelman H, Trijbels JMF, Sengers RCA, Janssen AJM. Measurements of cytochromes in human skeletal muscle mitochondria, isolated from fresh and frozen stored muscle specimens. Biochem Med 1978; 19: 366-73.

7 Lowry OH, Rosenbrough NJ, Farr AL, Randall RL. Protein measurement with the folin phenol reagent. J Biol Chem 1951; 193: $265-75$.

8 Johnson MA. Skeletal muscle. In: Filipe MI, Lake BD, eds. Histochemistry in pathology. Edinburgh: Churchill Livingstone, 1983: 89-113.

9 Neville HE, Ultrastructural changes in diseases of human skeletal muscle. In: Vinken PJ, Bruyn GW, eds. Handbook of clinical neurology. Amsterdam: North-Holland, 1979:40: 63-123.

10 van Senus AHC. Leber's disease in the Netherlands. Doc Ophthalmol 1963: 17: 1-162.

11 Yamanaka H. The mode of inheritance of Leber's discase. Acta Soc Ophthalmol Jpn 1971; 75 : 1930-6.

12 Ohba N, Tanino T, Izawa Y, Yoshida K. A pedigrec of Leber's optic atrophy. Jpn J Clin Ophthalmol 1977; 70: 56-8.

13 Egger J, Wilson J. Mitochondrial inheritance in a mitochondrially mediated disease. N Engl J Med 1983; 309: 142-6.

14 Merril CR. Harrington MG. The scarch for mitochondrial inheritance of human diseases. Trends Genet 1985; 1: 140-4.

15 Sengers RCA. Stadhouders AM, Trijbels JMF. Mitochondrial myopathies. Eur J Pediatr 1984; 141: 192-207.

16 DiMauro S, Bonilla E, Zeviani M, Nakagawa M, DeVivo DC. Mitochondrial myopathies. Ann Neurol 1985; 17: 521-35.

17 Nikoskclainen E, Paljärvi L, Lang H, Kalimo H. Leber's hereditary optic neuropathy: a mitochondrial discase? Acta Neurol Scand 1984; 69: 172-3.

18 Cagianut B. Rhyner K. Furrer W. Schncbli HP. Thiosulphatesulphur transferase (rhodanese) deficiency in Leber's hereditary optic atrophy. Lancet 1981; ii: 981-2.

Accepted for publication 22 August 1986. 